\title{
Transient increase in CSF GAP-43 concentration after ischemic stroke
}

\author{
Åsa Sandelius ${ }^{1,9^{*}}$ (D), Nicholas C. Cullen ${ }^{1,2}$, Åsa Källén ${ }^{3}$, Lars Rosengren ${ }^{4}$, Crister Jensen ${ }^{5}$, Vesna Kostanjevecki ${ }^{6}$, \\ Manu Vandijck ${ }^{6}$, Henrik Zetterberg ${ }^{1,3,7,8}$ and Kaj Blennow ${ }^{1,3,9^{*}}$
}

\begin{abstract}
Background: Cerebrospinal fluid (CSF) biomarkers reflect ongoing processes in the brain. Growth-associated protein 43 (GAP-43) is highly upregulated in brain tissue shortly after experimental ischemia suggesting the CSF GAP-43 concentration may be altered in ischemic brain disorders. CSF GAP-43 concentration is elevated in Alzheimer's disease patients; however, patients suffering from stroke have not been studied previously.

Methods: The concentration of GAP-43 was measured in longitudinal CSF samples from 28 stroke patients prospectively collected on days $0-1,2-4,7-9,3$ weeks, and 3-5 months after ischemia and cross-sectionally in 19 controls. The stroke patients were clinically evaluated using a stroke severity score system. The extent of the brain lesion, including injury size and degrees of white matter lesions and atrophy were evaluated by CT and magnetic resonance imaging.

Results: Increased GAP-43 concentration was detected from day 7-9 to 3 weeks after stroke, compared to day 1-4 and to levels in the control group $(P=0.02$ and $P=0.007)$. At 3-5 months after stroke GAP-43 returned to admission levels. The initial increase in GAP-43 during the nine first days was associated to stroke severity, the degree of white matter lesions and atrophy and correlated positively with infarct size $\left(r_{\mathrm{s}}=0.65, P=0.001\right)$.

Conclusions: The transient increase of CSF GAP-43 is important to take into account when used as a biomarker for other neurodegenerative diseases such as Alzheimer's disease. Furthermore, GAP-43 may be a marker of neuronal responses after stroke and additional studies confirming the potential of CSF GAP-43 to reflect severity and outcome of stroke in larger cohorts are warranted.
\end{abstract}

Keywords: GAP-43, Stroke, Neurodegeneration, Cerebrospinal fluid, Biomarkers

\section{Background}

The presynaptic protein Growth associated protein 43 (GAP-43) is highly expressed during neuronal development and synaptogenesis and thereafter sustained in presynaptic terminals in the hippocampus and associate cortex in the adult human brain [1-3]. GAP-43 is involved in the regulation of axonal outgrowth, synaptic plasticity, and learning and memory functions [4-9]. Considering the function of GAP-43, it may also be induced by neuronal damage caused by stroke, traumatic brain injury and epilepsy [8, 10-14]. Highly elevated levels of GAP-43 protein in the peri-infarct region after

\footnotetext{
* Correspondence: sandelius.asa@gmail.com; kaj.blennow@neuro.gu.se ${ }^{1}$ Institute of Neuroscience and Physiology, Department of Psychiatry and Neurochemistry, The Sahlgrenska Academy at the University of Gothenburg, Mölndal, Sweden

Full list of author information is available at the end of the article
}

experimentally induced cerebral ischemia have been well documented in rodents [11, 14-17]. Additionally, in rodent stroke models, a rapid induction of both GAP-43 gene and protein expression in the brain is reportedly present already at day one after ischemia and sustained up to 28 days and GAP- 43 was suggested to be an early and sensitive marker of neuronal damage after ischemia $[11,17,18]$. However, GAP-43 quantification has to our knowledge not been measured in any body fluids of stroke patients. Novel stroke biomarkers could improve clinical diagnosis, predict prognosis, and guide therapeutic interventions. Furthermore, even though CSF GAP-43 concentration could become useful as a biomarker of neuronal injury after stroke, it is equally important to examine confounding conditions that may affect the use of CSF GAP-43 as a biomarker or outcome measurement in other

(c) The Author(s). 2018 Open Access This article is distributed under the terms of the Creative Commons Attribution 4.0 International License (http://creativecommons.org/licenses/by/4.0/), which permits unrestricted use, distribution, and 
neuronal diseases. We evaluated CSF GAP-43 concentration in longitudinally sampled stroke patients to 1 ) further determine the pathogenic mechanisms of increased CSF GAP-43 concentration, and 2) investigate the biomarker potential of CSF GAP-43 after stroke.

\section{Methods}

\section{Study participants and sample collection}

The study was approved by the ethics committee for medical research at the University of Gothenburg, Sweden. Longitudinal CSF samples were collected prospectively from 28 patients with an acute ischemic stroke that occurred within the first 3 days of admission at Sahlgrenska University Hospital, Department of Neurology, between September 1992 and January 1994. Exclusion criteria were patients with a history of previous stroke, malignant or autoimmune diseases, severe infections, or taking immunosuppressive drugs. When possible, CSF samples were collected on up to five occasions; at admission to the emergency ward at day $0-1$, and then day $2-4$, day 7-9, 3 weeks and 3-5 months. CSF samples were taken by lumbar puncture in the L3/L4 or L4/L5 interspace, the first $12 \mathrm{ml}$ of CSF was collected in polypropylene tubes, centrifuged at $2000 \mathrm{~g}$ for $10 \mathrm{~min}$, and stored at $80^{\circ} \mathrm{C}$ pending biochemical analyses.

All stroke patients were clinically evaluated in a standardized way, as described previously [19]. Briefly, stroke severity was evaluated using a modified Scandinavian Stroke Scale Index (SSI) [19, 20]. The modified SSI is built on clinical scoring of seven parameters (between 1 and 5): consciousness, speech, facial paralysis, gait and physical strength in hand, arm and leg. In the modified version the maximum score is 27 and a higher score indicates increased severity of symptoms. Brain atrophy and infarct size was evaluated by magnetic resonance imaging (MRI) and computed tomography (CT) as previously described [19]. Atrophy in patients was scored as normal $(n=9)$, mild $(n=8)$, moderate $(n=4)$, and pronounced $(n=3)$, while white matter lesion status was scored as normal $(n=15)$, general-light $(n=1)$, general-moderate $(n=3)$, and cortical-light $(n=5)$ on T2-weighted MRI scans. Two patients lacked information about atrophy and white matter lesion, and two additional patients did not have enough samples for longitudinal analysis. Therefore, there were 24 patients for analysis of associations between these clinical measures and GAP-43 concentration. The control group consisted of healthy volunteers, without history, symptoms or signs of cognitive disturbances, neurological or psychiatric symptoms.

\section{Cerebrospinal fluid growth associated protein 43 enzyme- linked immunosorbent assay}

CSF GAP-43 concentration was measured by an in house enzyme-linked immunosorbent assay (ELISA) described in detail previously [21], with minor modifications. Microwell modules (Thermo Fisher Scientific, Massachusetts, USA) were coated with a mouse anti-GAP-43 antibody $(1.35 \mu \mathrm{g} /$ ml NM4, Fujirebio, Ghent, Belgium) in carbonate buffer $\mathrm{pH} 9.6$, overnight at $4{ }^{\circ} \mathrm{C}$. After washing in $0.05 \%$ Tween/ phosphate buffered saline (PBST), wells were blocked with $2 \%$ non-fat milk/PBST (assay diluent) for $1 \mathrm{~h}$ at room temperature, frozen at $-20^{\circ} \mathrm{C}$ until further use. After additional washes, in-house recombinant full length GAP-43 calibrators $(78 \mathrm{pg} / \mathrm{ml}-5000 \mathrm{pg} / \mathrm{ml})$, blanks, control samples and CSF samples pre-diluted 1:2 in assay diluent were co-incubated with a rabbit detector antibody $(0.20 \mu \mathrm{g} / \mathrm{ml}$ ABB-135, Nordic Biosite, Täby, Sweden) overnight at $4{ }^{\circ} \mathrm{C}$. Then, plates were washed and incubated with anti-rabbit HRP (1:20000, IgG $(\mathrm{H}+\mathrm{L}$, Cross-adsorbed Secondary Antibody horse radish peroxidase, ThermoFisherScientific, USA) for $2 \mathrm{~h}$ in $1 \%$ bovine serum albumin/PBST. After subsequent washes, wells were incubated for $30 \mathrm{~min}$ with 3,3',5,5'-tetramethylbenzidine (KemEnTech Diagnostics) in dark. The color reaction was stopped by addition of $0.2 \mathrm{M} \mathrm{H}_{2} \mathrm{SO}_{4}$ and the absorbance was read in a Sunrise microplate absorbance reader (Tecan group, Männedorf, Switzerland) at $450 \mathrm{~nm}(650 \mathrm{~nm}$ as reference value). Cerebrospinal fluid sample concentration was calculated via interpolation from the calibrator curve (4PL weighted $1 / \mathrm{Y} 2$ ).

\section{Assay validation}

For CSF GAP-43 assay characterization, intra- and inter-assay precision, measurement range, recovery, parallelism, selectivity and sample storage stability was evaluated. For intra- and inter-assay precision, five duplicates of two samples with high and low concentrations, respectively, were analyzed on 5 different days. Two technicians were involved in the acquisition of data. For determination of measurement range (lower limit of quantification (LLOQ) and upper limit of quantification (ULOQ)), calibration curve data from 5 runs were used. The relative error of the back-calculated concentrations for the calibrators was plotted as a function of concentration, and LLOQ and ULOQ determined as calibrator points with $<20 \%$ relative errors. Parallelism was determined in duplicates of one sample analyzed as neat and diluted $\times 2, \times 4, \times 8, \times 16$ and $\times 32$ times in assay diluent. Recovery was determined in two samples spiked with the GAP-43 calibrator. Neurogranin interference was determined in duplicates of one neat sample and the same with $10 \mathrm{ng} / \mathrm{ml}$ recombinant neurogranin protein spike was measured. Protein stability was evaluated by dividing 3 samples into nine aliquots where one was directly placed at $-80^{\circ} \mathrm{C}$, and the others stored at $-20^{\circ} \mathrm{C}$ or at $4{ }^{\circ} \mathrm{C}$ overnight or for 1 week, at room temperature for $24 \mathrm{~h}$, or freeze-thawed 1-4 times.

\section{Statistical analysis}

Because the longitudinal trajectory of GAP-43 values appeared to be nonlinear, the data was initially analyzed in 
two parts. First, a linear mixed effects model was fit on measurements taken between before 9 days to test the hypothesis that GAP-43 increases directly after stroke. The model included random intercepts and slopes, and time was discretized into Days 0-1, Days 2-3, and Days 7-9. Age and sex were included as covariates and GAP-43 concentrations were normalized for each individual relative to their baseline measurement. Next, the same form of mixed model was fit on measurements taken from Days 7-9, 2-4 weeks, and 3-5 months in order to test the hypothesis that GAP-43 returns to normal levels over time. Differences in GAP-43 concentration across groups were analyzed using approximate $t$ tests on the estimated marginal means. The Pearson correlation coefficient was calculated between subject-specific slopes for the first model of early measurements and the subject-specific slopes of the second model of late measurements to understand the relationship between initial change and late change in GAP-43 concentrations.

A linear mixed model was then fit on longitudinal stroke severity information, again with random intercepts and slopes. The relationship between GAP-43 change and stroke severity change was subsequently assessed by calculating the Pearson correlation coefficient between the subject-specific slopes of stroke severity and subject-specific slopes of GAP-43 as estimated from the respective mixed effects models. The association between GAP-43 change and infarct size was also computed using the Pearson correlation coefficient.

Additionally, group differences in GAP-43 change across discrete levels of white matter lesions and across discrete levels of brain atrophy were analyzed by fitting the same linear mixed effects model as above but with white matter lesion (or brain atrophy) status included as an interaction with time. Overall group difference was assessed using approximate $F$ tests and between each pair of groups using approximate $t$ tests on the estimated marginal means. Finally, concentrations of GAP-43 in healthy controls were compared to those of stroke patients at each time group using Mann-Whitney U tests, with correction for multiple comparisons using Holm's procedure.

All tests were two-sided with a significance level set to $p=0.05$. All statistical analysis was performed using the $\mathrm{R}$ programming language (version 3.4.3) with the nlme (version 3.1) and emmeans (version 1.2) packages being used for mixed effects modelling specifically.

\section{Results}

\section{Patient demographics}

Demographics of study participants are provided in Table 1. The control group was age matched to the clinical stroke group although with a larger percentage of females. There was no correlation between age and CSF GAP-43 concentration in stroke or control groups $\left(r_{\mathrm{s}}(\right.$ day $0-4)=0.43, p=0.13$; control: $r_{\mathrm{s}}=-0.23, p=0.34$ ) and no differences between gender (stroke (day 0-4): $p=0.78$; control: $p=0.45$ ). For white matter lesion status in the stroke patients, there were nine individuals who scored as normal, eight as sporadic, four as moderate, and three as pronounced. For brain atrophy, 15 individuals were scored as normal, one as general-light, three as general-moderate, and five as cortical-light.

\section{Assay validation results}

The average intra- and inter-assay variation was assessed by determining the coefficient of variation $(\mathrm{CV})$ of repeated analysis. The intra- and inter-assay CVs were 3.4 and $7.4 \%$ respectively, for a sample with a mean GAP-43 concentration of $1184 \mathrm{pg} / \mathrm{ml}$, and 3.2 and $5.8 \%$ respectively, for a sample with a mean GAP-43 concentration of $3117 \mathrm{pg} / \mathrm{ml}$. The LLOQ was $315 \mathrm{pg} / \mathrm{ml}$ and ULOQ was $10,000 \mathrm{pg} / \mathrm{ml}$. The parallelism was $85-100 \%$ for samples diluted $2-8$ times. Recovery was $99-109 \%$. No neurogranin interference was detected (spike sample $(1751 \mathrm{pg} / \mathrm{ml})$; GAP-43 recovery was $101 \%$ of neat sample $(1734 \mathrm{pg} / \mathrm{ml}))$. All stability aliquots were analyzed simultaneously and their concentrations differed between 81 and $116 \%$ for samples with different storage conditions, and $75-115 \%$ for freeze-thaw cycled aliquots, compared to the original sample stored at $-80^{\circ} \mathrm{C}$.

\section{CSF GAP-43 concentration increases directly after stroke and eventually returns to initial levels}

CSF GAP-43 concentrations in controls and change over time in stroke patients are depicted in Fig. 1. GAP-43 concentration increased significantly over time relative to baseline in the first 9 days after stroke (relative change: $+19 \%$ per day, 95\% CI [11.1, 26.9]; $\mathrm{F}_{1,32}=24.8, P<0.0001$, Fig. 1a). This result also held when viewing GAP-43 in absolute terms (absolute change: $+506.6 \mathrm{pg} / \mathrm{mL}$ per day, 95\% CI [204.0, 809.3], $\mathrm{F}_{1,32}=11.7, P=0.002$, Fig. 1b). Additionally, GAP-43 concentrations between Days 7-9 were significantly elevated compared to Days $0-1$ (relative difference: $86.4,95 \%$ CI $[11.8,161.0] ; \mathrm{t}_{31}=2.4, P=0.02$, Fig. 1a) and compared to Days 2-3 (relative difference: $74.5,95 \%$ CI $[15.8,133.4]$; $\mathrm{t}_{31}=2.6, P=0.02$, Fig. 1a).

Additionally, GAP-43 concentration decreased significantly starting 9 days after stroke relative to baseline (relative change: $-0.6 \%$ per day, $95 \% \mathrm{CI}[0.3,1.2] ; \mathrm{F}_{1,38}=4.5, P=0.04$ ) and absolute GAP-43 concentrations also decreased significantly over the same period (absolute change: $-30.2 \mathrm{pg} / \mathrm{mL}$ per day, $95 \%$ CI $[-5.7,-54.7] ; \mathrm{F}_{1,38}=6.2, P=0.02$ ). Additionally, GAP-43 concentrations between 3 and 5 months were significantly lower compared to 3 weeks (relative difference: $86.1,95 \%$ CI $[2.1,170.2] ; \mathrm{t}_{40}=2.6$, $P=0.04$ ) and compared to Days 7-9 (relative difference: $81.3,95 \%$ CI $\left.[-4.2,166.8] ; \mathrm{t}_{40}=2.4, P=0.04\right)$.

Overall, GAP-43 concentration increased slightly relative to baseline (mean: 100\%) in Days 2-3 (mean: 118.1\%) and increased greatly in Days 7-9 (mean: $187.1 \%$ ), then began to decrease relative to Days 7-9 after 3 weeks (mean: 166.6\%) 
Table 1 Study participant demographics, clinical and biochemical data

\begin{tabular}{|c|c|c|}
\hline & Controls & Stroke patients \\
\hline \multicolumn{3}{|l|}{ Demographics } \\
\hline No. & 19 & 28 \\
\hline Age & $66( \pm 7)$ & $64( \pm 11)$ \\
\hline Gender (\% male) & $47 \%$ & $75 \%$ \\
\hline SSI $0<15<30(n)$ & & $23 / 5 / 0$ \\
\hline White matter lesion: normal/sporadic/moderate/ pronounced (n) & & $9 / 8 / 4 / 3$ \\
\hline Atrophy: normal/ general sporadic/general moderate/cortical sporadic (n) & & $15 / 1 / 3 / 5$ \\
\hline GAP-43 (pg/ml) & $2711(2021-3602)$ & \\
\hline Day $0-1(n=8)$ & & $2008(1762-2954)$ \\
\hline Day2-4 $(n=23)$ & & $3311(2057-5491)$ \\
\hline Day7-9 $(n=26)$ & & $3819(2531-7242)$ \\
\hline 3 weeks $(n=22)$ & & $3949(2668-6606)$ \\
\hline $3-5$ months $(n=24)$ & & $2562(2112-2821)$ \\
\hline
\end{tabular}

and returned to initial levels after 3-5 months (mean: 89.8\%). Continuous individual longitudinal trajectories of absolute GAP-43 concentration after stroke are shown in Fig. 1b, where each line represents a single individual (right of dotted line), showing that most stroke suffering individuals returned to control levels (Fig. 1b). The initial increase in GAP-43 concentration before 9 days was highly correlated with the subsequent decrease in GAP-43 levels over time ( $r=-0.65, P=0.0005$; see Fig. $1 c)$, indicating that the individuals with the largest initial increases in GAP-43 were more likely to have larger subsequent decreases in GAP-43 concentration later on.

Compared to cross-sectional measurements of healthy controls, absolute GAP-43 concentration in stroke patients was significantly elevated in Days 7-9 (median difference: 1628 pg/ $\mathrm{mL}, 95 \%$ CI [333, 3905]; $P=0.007$, Fig. 1d) and 3 weeks after stroke (median difference: $1115 \mathrm{pg} / \mathrm{mL}, 95 \% \mathrm{CI}$ [106, 2467]; $P=0.02$, Fig. 1d), but not in Days $0-1$ (median difference: $-207 \mathrm{pg} / \mathrm{mL}, 95 \%$ CI [-1252, 537]; $P=0.58$, Fig. 1d), Days 2-3 (median difference: $270 \mathrm{pg} / \mathrm{mL}, 95 \%$ CI [-390, 1388]; $P=0.44$, Fig. 1d), or $3-5$ months (median difference: $-128 \mathrm{pg} / \mathrm{mL}, 95 \%$ CI $[-774,567] ; P=0.73$, Fig. 1d) after stroke.

\section{Association between GAP-43 change and stroke severity,} white matter lesions, brain atrophy and infarct size

In the first 9 days after stroke, the increase in GAP-43 concentration was significantly associated with change in stroke severity as measured by the modified SSI scale ( $r=0.49, P=$ 0.01 , Fig. 2a). This association was strengthened when considering only those patients whose SSI scores improved over time $(r=0.82, P=0.002)$. However, there was no correlation between the change in GAP-43 concentration over time and the change of stroke severity $(r=-0.05, P=0.84$, Fig. $2 b)$ in the time period after 9 days.
The change in GAP-43 concentration until 9 days after stroke significantly differed overall across white matter lesion status $\left(\mathrm{F}_{3,27}=4.1, P=0.017\right)$, but not for change in GAP-43 concentration later on $\left(F_{3,33}=0.61, P=0.61\right)$. However, there was no significant difference between GAP-43 changes for any two specific white matter lesion groups likely due to lack of statistical power (see Additional file 1 for $\mathrm{F}$ and $P$-values).

Additionally, the change in GAP-43 concentrations up to 9 days after stroke again significantly differed across brain atrophy status $\left(\mathrm{F}_{3,26}=6.1, P=0.003\right)$, but not for GAP-43 concentrations after 9 days $\left(F_{3,31}=0.14, P=0.93\right)$. Again, there was no significant difference between GAP-43 changes for any two specific brain atrophy groups likely due to lack of statistical power (see Additional file 1 for F and P-values).

The initial increase in GAP-43 concentration over the first 9 days correlated positively with infarct size $(r=0.75$, $P<0.0001$ ), indicating that those with higher infarct size also had larger initial increase in GAP-43 levels. Meanwhile, the later decrease in GAP-43 concentration after 9 days correlated negatively with infarct size $(r=-0.64, P=0.001)$.

\section{Discussion}

In this study, we detected a transient increase in CSF GAP-43 concentration after acute ischemic stroke that was most prominent during the first 2 weeks after injury. As CSF is in direct contact with the brain, its content can reflect ongoing processes in the brain tissue. Animal models of ischemia suggest that the content of GAP-43 in the brain may be the result of both an increase due to regeneration responses and a decrease due to disrupted axons at the site of injury $[11,17,18]$. The temporary increase in CSF GAP-43 in our study likely reflects an increase due to regenerative responses, however additive effects of leakage of this synaptic protein from damaged neurons cannot be ruled out. Substantial axonal sprouting 

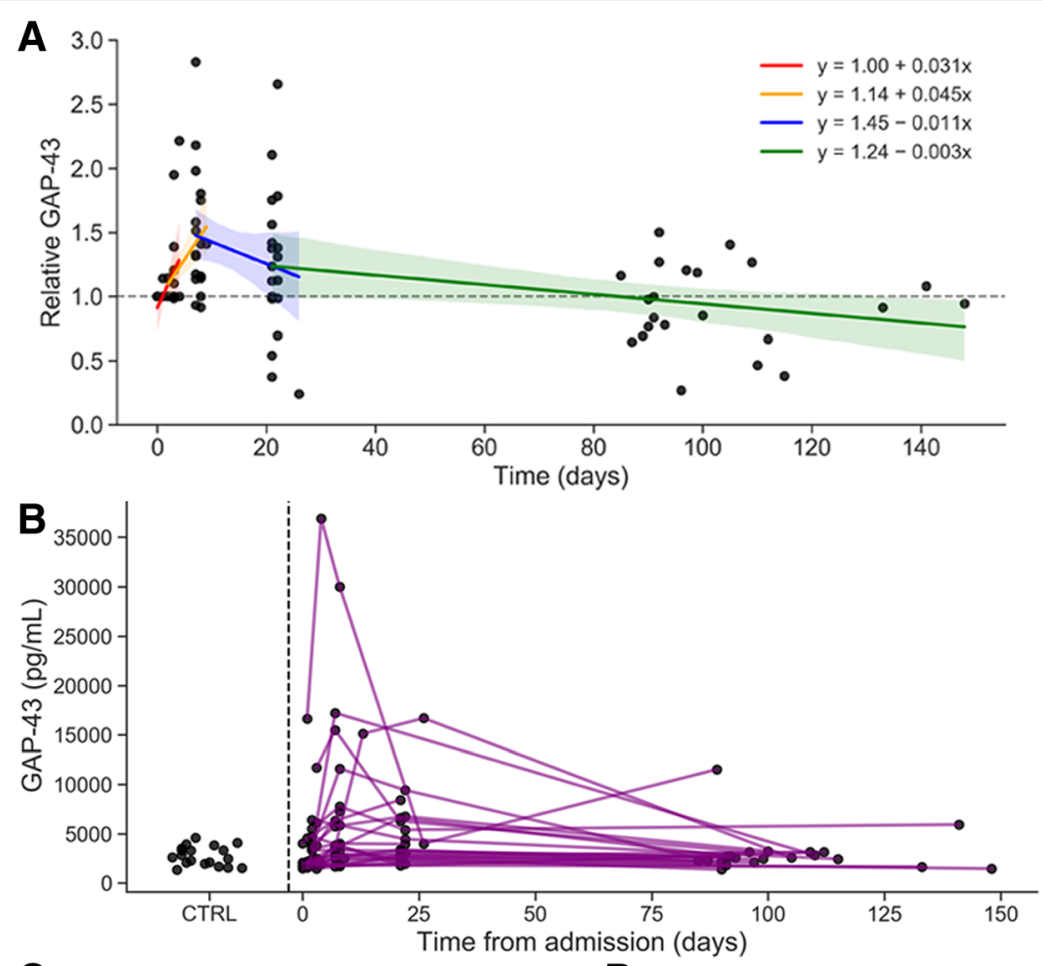

C

D

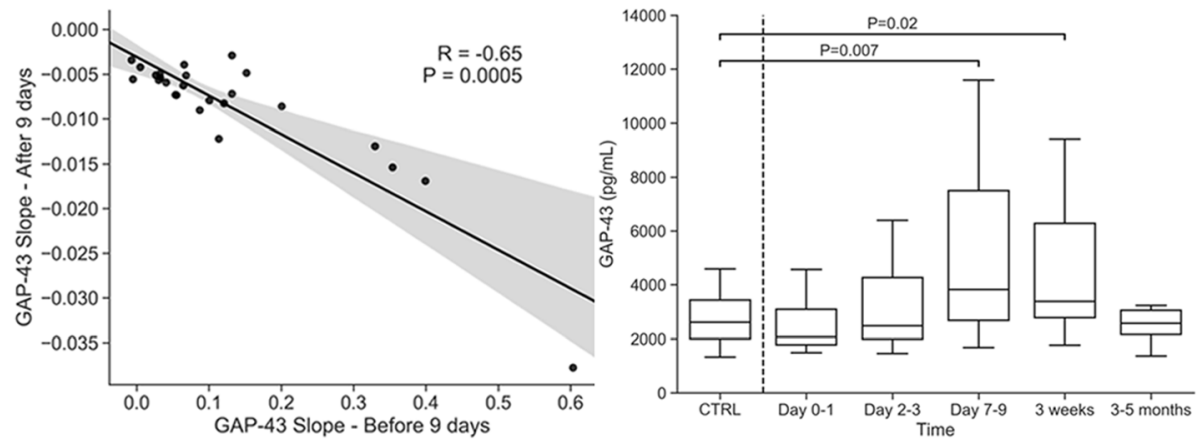

Fig. 1 Transient increase in CSF GAP-43 concentration after stroke. a GAP-43 concentration relative to each individual's baseline and a piecewise-linear regression model displaying overall longitudinal trends between the five time points; $\mathbf{b}$ Baseline GAP-43 levels in controls (left of dotted line) and continuous individual longitudinal trajectories of absolute GAP-43 concentration after stroke, where each line represents a single individual (right of dotted line), showed a transient increase directly after stroke which returned to control levels for most individuals; $\mathbf{c}$ Relationship between the increase in GAP-43 concentration before 9 days and the subsequent decrease in GAP-43 concentration after 9 days; $\mathbf{d}$ GAP-43 concentration (median and interquartile range) in controls (left of dotted line) and in stroke patients at Day 0-1, Day 2-3, Day 7-9, 3 weeks and 3-5 months

and cortical reorganization is initiated after stroke and reported in patients and experimental stroke models [22-26].

The CSF GAP-43 concentration in control subjects was similar to most stroke patients at admission (Day 0-1, Day 23) and the following increase during the first 9 days after stroke indicate that increased CSF GAP-43 results from a delayed or progressing process. Different mechanisms of ischemia-induced cell death are acutely activated in the ischemic core, appearing within a time-window of $0-2$ days [27]. In addition, delayed neuronal death is known to appear several days after ischemia and progressing weeks after the injury $[28$,
29]. The delayed increase of GAP-43 in CSF may reflect the time it takes for neuronal injury to spread across larger peri-infarct areas, or reflect the time it takes for GAP-43 expressing neurons to die, with release of GAP-43 to the extracellular space and CSF. This is further supported by a study reporting decreased numbers of GAP-43 expressing cortical cells in post-ischemic patients [30]. Also, GAP-43 expressing neurons may be more resistant to the direct ischemic event. It has been suggested that different types of neurons are differently susceptible to early and delayed cell death after ischemic injuries [31]. 

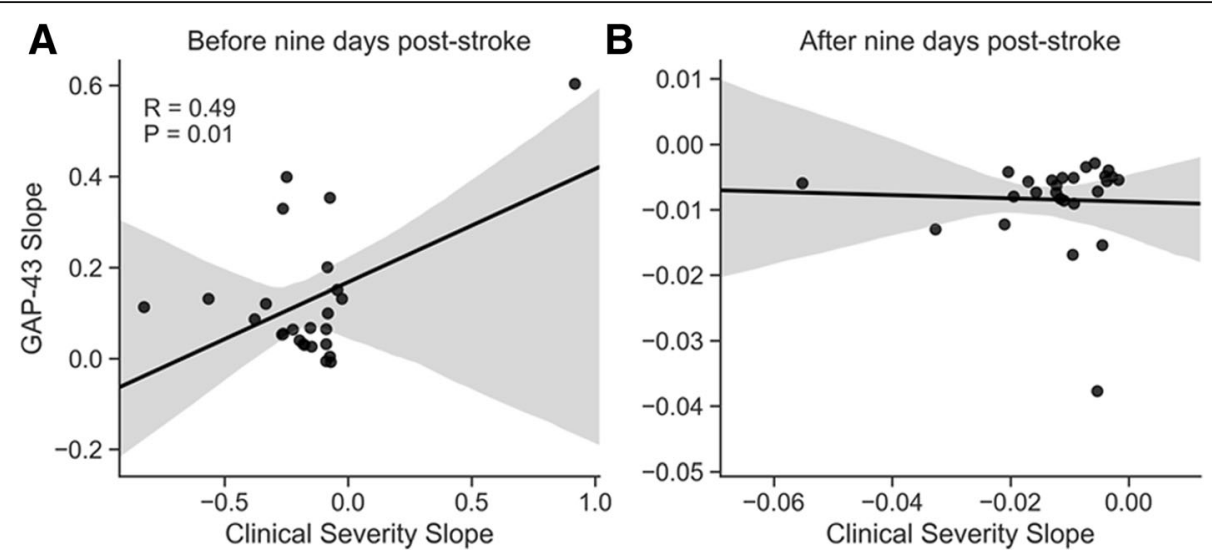

Fig. 2 Association of CSF GAP-43 to clinical characteristics. a Relationship between increase in GAP-43 concentration and the worsening of clinical stroke severity score in the period until 9 days after stroke; $\mathbf{b}$ the same as panel a, but for the period after 9 days after stroke

Moreover, our results suggest an association of CSF GAP-43 concentration and clinical characteristics during the first 2 weeks after ischemia. Larger increase in CSF GAP-43 was related not necessarily to a worsening in clinical characteristics but rather a lack of improvement, given that all but one individual either improved or stayed the same over the course of the study. This connection was even stronger when analyzing only those patients whose clinical characteristics actually improved. The connection to white matter lesions indicates that GAP-43 levels were likely to increase more in individuals with pronounced white matter lesions. Similarly, a high increase in CSF GAP-43 concentration was related to more severe atrophy and larger infarct size. Together with weaker, or the lack of, association with clinical measures at later time points, this suggests that CSF GAP-43 concentration primarily reflect the degree of, or response to, neuronal injury but not recovery. Limitations of this study include the relatively low sample number and the rather long sample storage time. The reason for the long sample storage time is that a stable CSF GAP-43 quantification method was just recently developed and this evaluation of CSF GAP-43 in longitudinal stroke samples was possible through a previous established collaboration. However, longitudinal studies have the advantage of letting each patient be its own control at baseline and individual changes over time should not be considerably affected assuming equal effects of storage on all samples. An additional limitation is the use of the modified version of the SSI, which is not standardized internationally. Nonetheless, we show for the first time that changes in CSF GAP-43 after stroke are connected to clinical measures. Additional studies are required to further evaluate the value of GAP-43 as a stroke biomarker and elucidate its connections to injury progression and clinical outcome. CSF GAP-43 could become useful as a quantitative measure of the regenerative response during recovery, which should simplify comparison and evaluation of both stroke treatments and rehabilitation strategies.

Importantly, the results reported here, demonstrating a clear effect of ischemia on CSF GAP-43 concentrations, need to be considered when using CSF GAP-43 as a biomarker in other settings. In a previous study of several dementia disorders, we have shown a specific increase in CSF GAP-43 in Alzheimer's disease compared to several other neurodegenerative diseases [21]. As increased CSF GAP-43 concentration was associated to Alzheimer's disease neuropathology and correlated with cognitive decline, it could be useful as an outcome marker in clinical trials for novel Alzheimer's disease therapeutics. As stroke is likely to occur in a fraction of the study population during a drug trial period, it would be important to take into consideration that CSF GAP-43 is transiently increased after stroke affecting outcome levels. Other CSF biomarkers such as t-tau, p-tau and neurogranin have also been evaluated in stroke as well as traumatic brain injury to elucidate mechanisms of neuronal injury reflected by particular biomarkers [32-36]. It was found that t-tau, but not $\mathrm{p}$-tau, increased after stroke suggesting they reflect different disease/injury mechanisms [33, 36]. Similar to our findings, CSF t-tau and neurogranin concentrations were related to stroke characteristics $[32,34]$. Although CSF GAP-43 was previously found largely selective for Alzheimer's disease among neurodegenerative diseases, it may be altered by yet other conditions besides stroke. Changes of GAP-43 expression levels in experimental models of epilepsy and traumatic brain injury urges for additional investigations of CSF GAP-43 change in epilepsy and traumatic brain injury patients to find out the potential use of GAP-43 concentration as a neuronal injury biomarker. 


\section{Conclusion}

The transient increase of CSF GAP-43 appearing most intensely at 1-3 weeks after stroke is important to take into account when used as a biomarker for other neurodegenerative diseases. Our findings also suggest that CSF GAP-43 may be a marker of neuronal injury responses in stroke and urge for additional studies confirming the potential of CSF GAP-43 to reflect severity and outcome of stroke in larger cohorts.

\section{Additional file}

Additional file 1: Table of $t$ and $p$ values across the different white matter lesion status and brain atrophy status groups, up to 9 days and, after 9 days past the stroke. (XLSX 9 kb)

\section{Abbreviations}

CSF: Cerebrospinal fluid; CT: Computed tomography; ELISA: Enzyme-linked immunosorbent assay; GAP-43: Growth associated protein 43; LLOQ: Lower limit of quantification; MRI: Magnetic resonance imaging; SSI: Scandinavian Stroke Scale Index; ULOQ: Upper limit of quantification

\section{Acknowledgements}

Not applicable.

\section{Funding}

The authors are thankful to Gamla Tjänarinnor Foundation, Demensfonden, Sigurd och Elsa Golje Foundation and Stiftelsen Edit Jacobsons Donationsfond for funding. The study was further supported in part by the Swedish Research Council, the European Research Council, the Knut and Alice Wallenberg Foundation, the Torsten Söderberg Foundation and Swedish State Support for Clinical Research (ALFGBG). The funders had no role in the design of the study and collection, analysis, interpretation of data or in writing the manuscript.

\section{Availability of data and materials}

The datasets used and/or analyzed during the current study are available from the corresponding author on reasonable request.

\begin{abstract}
Authors' contributions
Ås developed and validated the ELISA, analyzed and interpreted results and wrote the manuscript. NCC performed statistical analysis and was involved in drafting the manuscript. ÅK generated data and made critical revision of the manuscript. LR prepared the study layout and performed clinical evaluation of patients, interpreted data, and was a major contributor in writing the manuscript. CR performed the $C T$ and MRI and made critical revision of the manuscript. VK performed method development and made critical revision of the manuscript. MV performed method development and made critical revision of the manuscript. $\mathrm{HZ}$ analyzed and interpreted data, and was a major contributor in writing the manuscript. KB analyzed and interpreted data, and was a major contributor in writing the manuscript. All authors read and approved publication of the final manuscript and agreed to be accountable for all aspects of the work in ensuring that questions related to the accuracy or integrity of any part of the work are appropriately investigated and resolved.
\end{abstract}

\section{Ethics approval and consent to participate}

Prior to inclusion, informed consent was obtained from each patient. All were included into the study by one of the authors (LR). All asked were alert (RLS 1; [37]) and without impressive aphasia. In all cases, consent was obtained directly from the patient. In many cases the patient conferred with family members but they were never advised to decline participation. Patients were informed by verbal and written information and consent was given in verbal form, because at the time for ethical approval (15th of April 1992) the ethical committee did not yet require written consent or raise the question. The criteria were approved by the Gothenburg Regional Ethics Committee (Dnr 172-92), Sweden.

\section{Consent for publication}

Not applicable.

\section{Competing interests}

$\mathrm{HZ}$ is a co-founder of Brain Biomarker Solutions in Gothenburg AB, a GU Ventures-based platform company at the University of Gothenburg, has served at advisory boards of Eli Lilly, Roche Diagnostics, Wave, Samumed and CogRx and has received travel support from TEVA.

KB has served as a consultant or at advisory boards for Alzheon, BioArctic, Biogen, Eli Lilly, Fujirebio Europe, IBL International, Merck, Pfizer, and Roche Diagnostics, and is a co-founder of Brain Biomarker Solutions in Gothenburg $A B$, a GU Ventures-based platform company at the University of Gothenburg. Other authors declare that they have no competing interests.

\section{Publisher's Note}

Springer Nature remains neutral with regard to jurisdictional claims in published maps and institutional affiliations.

\section{Author details}

${ }^{1}$ Institute of Neuroscience and Physiology, Department of Psychiatry and Neurochemistry, The Sahlgrenska Academy at the University of Gothenburg, Mölndal, Sweden. ${ }^{2}$ Department of Neurology, Perelman School of Medicine, University of Pennsylvania, Philadelphia, USA. ${ }^{3}$ Clinical Neurochemistry Laboratory, Sahlgrenska University Hospital, Mölndal, Sweden. ${ }^{4}$ Institute of Neuroscience and Physiology, Department of Clinical Neuroscience and Rehabilitation, The Sahlgrenska Academy at University of Gothenburg, Gothenburg, Sweden. ${ }^{5}$ Institute of Clinical Sciences, University of Gothenburg, Gothenburg, Sweden. ${ }^{6}$ Fujirebio Europe nv, Ghent, Belgium. ${ }^{7}$ UK Dementia Research Institute, WC1N, London, UK. ${ }^{8}$ Department of Neurodegenerative Disease, UCL Institute of Neurology, Queen Square, London, UK. ${ }^{9}$ Department of Psychiatry and Neurochemistry, Sahlgrenska University Hospital/Mölndal, S-431 80 Mölndal, Sweden.

Received: 27 August 2018 Accepted: 29 November 2018

Published online: 07 December 2018

\section{References}

1. Neve RL, Finch EA, Bird ED, Benowitz LI. Growth-associated protein GAP-43 is expressed selectively in associative regions of the adult human brain. Proc Natl Acad Sci U S A. 1988:85(10):3638-42

2. De la Monte SM, Federoff HJ, Ng SC, Grabczyk E, Fishman MC. GAP-43 gene expression during development: persistence in a distinctive set of neurons in the mature central nervous system. Brain Res Dev Brain Res. 1989;46(2):161-8.

3. Riascos D, Nicholas A, Samaeekia R, Yukhananov R, Mesulam MM, Bigio EH, et al. Alterations of ca (2) (+)-responsive proteins within cholinergic neurons in aging and Alzheimer's disease. Neurobiol Aging. 2014;35(6):1325-33.

4. Aigner L, Arber S, Kapfhammer JP, Laux T, Schneider C, Botteri F, et al. Overexpression of the neural growth-associated protein gap-43 induces nerve sprouting in the adult nervous-system of transgenic mice. Cell. 1995;83(2):269-78.

5. Skene JHP, Jacobson RD, Snipes GJ, Mcguire CB, Norden JJ, Freeman JA. A protein-induced during nerve growth (gap-43) is a major component of growth-cone membranes. Science. 1986;233(4765):783-6.

6. Routtenberg A, Cantallops I, Zaffuto S, Serrano P, Namgung U. Enhanced learning after genetic overexpression of a brain growth protein. Proc Natl Acad Sci U S A. 2000;97(13):7657-62.

7. Holahan MR, Honegger KS, Routtenberg A. Ectopic growth of hippocampal mossy fibers in a mutated GAP-43 transgenic mouse with impaired spatial memory retention. Hippocampus. 2010;20(1):58-64.

8. Allegra Mascaro AL, Cesare P, Sacconi L, Grasselli G, Mandolesi G, Maco B, et al. In vivo single branch axotomy induces GAP-43dependent sprouting and synaptic remodeling in cerebellar cortex. Proc Natl Acad Sci U S A. 2013;110(26):10824-9.

9. Young EA, Owen EH, Meiri KF, Wehner JM. Alterations in hippocampal GAP43 phosphorylation and protein level following contextual fear conditioning. Brain Res. 2000;860(1-2):95-103.

10. Nemes AD, Ayasoufi K, Ying Z, Zhou QG, Suh H, Najm IM. Growth associated protein 43 (GAP-43) as a novel target for the diagnosis, treatment and prevention of Epileptogenesis. Sci Rep. 2017;7(1):17702.

11. Gorup D, Bohacek I, Milicevic T, Pochet R, Mitrecic D, Kriz J, et al. Increased expression and colocalization of GAP43 and CASP3 after brain ischemic lesion in mouse. Neurosci Lett. 2015:597:176-82.

12. Chong MS, Reynolds ML, Irwin N, Coggeshall RE, Emson PC, Benowitz LI, et al. Gap-43 expression in primary sensory neurons following central Axotomy. J Neurosci. 1994;14(7):4375-84. 
13. Hulsebosch CE, DeWitt DS, Jenkins LW, Prough DS. Traumatic brain injury in rats results in increased expression of gap-43 that correlates with behavioral recovery. Neurosci Lett. 1998;255(2):83-6.

14. Yamada K, Goto S, Oyama T, Inoue N, Nagahiro S, Ushio Y. In-vivo induction of the growth-associated protein Gap43/B-50 in rat astrocytes following transient middle cerebral-artery occlusion. Acta Neuropathol. 1994;88(6):553-7.

15. Stroemer RP, Kent TA, Hulsebosch CE. Acute increase in expression of growth associated protein GAP-43 following cortical ischemia in rat. Neurosci Lett. 1993;162(1-2):51-4.

16. Goto S, Yamada K, Inoue N, Nagahiro S, Ushio Y. Increased expression of growth-associated protein GAP-43/B-50 following cerebral hemitransection or striatal ischemic injury in the substantia nigra of adult rats. Brain Res. 1994;647(2):333-9.

17. Li Y, Jiang N, Powers C, Chopp M. Neuronal damage and plasticity identified by microtubule-associated protein 2, growth-associated protein 43, and cyclin D1 immunoreactivity after focal cerebral ischemia in rats. Stroke 1998;29(9):1972-80; discussion 80-1.

18. Carmichael ST, Archibeque I, Luke L, Nolan T, Momiy J, Li S. Growthassociated gene expression after stroke: evidence for a growth-promoting region in peri-infarct cortex. Exp Neurol. 2005;193(2):291-311.

19. Tarkowski E, Rosengren L, Blomstrand C, Wikkelso C, Jensen C, Ekholm S, et al. Early intrathecal production of interleukin-6 predicts the size of brain lesion in stroke. Stroke. 1995;26(8):1393-8.

20. Multicenter trial of hemodilution in ischemic stroke--background and study protocol. Scandinavian stroke study group. Stroke. 1985;16(5):885-90.

21. Sandelius A, Portelius E, Kallen A, Zetterberg H, Rot U, Olsson B, et al. Elevated CSF GAP-43 is Alzheimer's disease specific and associated with tau and amyloid pathology. Alzheimers Dement. 2018.

22. Dijkhuizen RM, Singhal AB, Mandeville JB, Wu O, Halpern EF, Finklestein SP, et al. Correlation between brain reorganization, ischemic damage, and neurologic status after transient focal cerebral ischemia in rats: a functional magnetic resonance imaging study. J Neurosci. 2003;23(2):510-7.

23. Wang L, Yu C, Chen H, Qin W, He Y, Fan F, et al. Dynamic functional reorganization of the motor execution network after stroke. Brain. 2010;133(Pt 4):1224-38.

24. Ward NS. Functional reorganization of the cerebral motor system after stroke. Curr Opin Neurol. 2004;17(6):725-30.

25. Kantak SS, Stinear JW, Buch ER, Cohen LG. Rewiring the brain: potential role of the premotor cortex in motor control, learning, and recovery of function following brain injury. Neurorehabil Neural Repair. 2012;26(3):282-92.

26. Carmichael ST, Kathirvelu B, Schweppe CA, Nie EH. Molecular, cellular and functional events in axonal sprouting after stroke. Exp Neurol. 2017;287(Pt 3):384-94.

27. Broughton BR, Reutens DC, Sobey CG. Apoptotic mechanisms after cerebral ischemia. Stroke. 2009;40(5):e331-9.

28. Nakano S, Kogure K, Fujikura H. Ischemia-induced slowly progressive neuronal damage in the rat brain. Neuroscience. 1990;38(1):115-24.

29. Pulsinelli WA, Brierley JB, Plum F. Temporal profile of neuronal damage in a model of transient forebrain ischemia. Ann Neurol. 1982;11(5):491-8.

30. Akulinin VA, Belichenko PV, Dahlstrom A. The cellular distribution of GAP-43 immunoreactivity in human neocortical areas using immunofluorescence and confocal microscopy: post-ischemic influence. Brain Res. 1998;784(1-2):341-6.

31. Chavez-Valdez R, Emerson P, Goffigan-Holmes J, Kirkwood A, Martin LJ, Northington FJ. Delayed injury of hippocampal interneurons after neonatal hypoxia-ischemia and therapeutic hypothermia in a murine model. Hippocampus. 2018;28(8):617-30.

32. De Vos A, Bjerke M, Brouns R, De Roeck N, Jacobs D, Van den Abbeele L, et al. Neurogranin and tau in cerebrospinal fluid and plasma of patients with acute ischemic stroke. BMC Neurol. 2017;17(1):170.

33. Hesse C, Rosengren L, Andreasen N, Davidsson P, Vanderstichele H, Vanmechelen E, et al. Transient increase in total tau but not phospho-tau in human cerebrospinal fluid after acute stroke. Neurosci Lett. 2001;297(3):187-90.

34. Ihle-Hansen H, Hagberg G, Fure B, Thommessen B, Fagerland MW, Oksengard AR, et al. Association between total-tau and brain atrophy one year after first-ever stroke. BMC Neurol. 2017;17(1):107.

35. Ost M, Nylen K, Csajbok L, Ohrfelt AO, Tullberg M, Wikkelso C, et al. Initial CSF total tau correlates with 1-year outcome in patients with traumatic brain injury. Neurology. 2006;67(9):1600-4.

36. Kaerst L, Kuhlmann A, Wedekind D, Stoeck K, Lange P, Zerr I. Cerebrospinal fluid biomarkers in Alzheimer's disease, vascular dementia and ischemic stroke patients: a critical analysis. J Neurol. 2013;260(11):2722-7.

37. Starmark JE, Stalhammar D, Holmgren E. The reaction level scale (RLS85). Manual and guidelines. Acta Neurochir (Wien). 1988;91(1-2):12-20.

Ready to submit your research? Choose BMC and benefit from:

- fast, convenient online submission

- thorough peer review by experienced researchers in your field

- rapid publication on acceptance

- support for research data, including large and complex data types

- gold Open Access which fosters wider collaboration and increased citations

- maximum visibility for your research: over $100 \mathrm{M}$ website views per year

At BMC, research is always in progress.

Learn more biomedcentral.com/submissions 